\title{
An apple- mango- egg- or potato a day?
}

\author{
Azra Rizwan', Farheen Dhojki', Faiza Shoeib ${ }^{1}$, Wajiha Afaq ${ }^{1}$, Qamar Masood ${ }^{1}$ and Zaman Sheikh ${ }^{2}$, Azra Rizwan ${ }^{1 *}$ \\ ${ }^{1}$ Aga Khan University Hospital [AKUH], Pakistan \\ ${ }^{2}$ National Institute Diabetes \& Endocrinology [NIDE] Dow University of Health Sciences, Karachi, Pakistan
}

\begin{abstract}
The purpose of this study was to determine dietary perceptions amongst adults with type 2 diabetes, to identify areas requiring emphasis during dietary teaching. We used questionnaire-based interviews to collect perspectives from 400 participants attending the Diabetes Clinic at a private and public hospital, Karachi, Pakistan. About one third of the subjects at both centers believed that fruit was prohibited for subjects with diabetes, with mangoes and bananas being most cited as the culprit fruits. Only $45 \%$ individuals from public sector, while $68 \%$ from the private sector were aware of the importance of whole wheat consumption versus refined grain. There was uncertainty regarding egg, meat, milk, and potato consumption. Several dietary misconceptions existed amongst diabetics, irrespective of socioeconomic or educational status. These results provide directions for development of culturally specific diabetes education and could create awareness amongst patients to progress from knowledge to implementation, possibly leading to improved diabetes control. Health care professionals need to keep themselves abreast with on-going research particularly regarding fruit, refined carbohydrate, and fat consumption in subjects with diabetes. This may help to remove myths and unnecessary dietary prohibitions placed on these already psychologically stressed individuals, with the tag of this metabolic aberration of dysglycemia, shaping their entire lives.
\end{abstract}

Doctor, what can I eat? Can I have fruit, but that is also "sweet". This question hounds every newly diagnosed subject with diabetes till this day. "Carrot and grapefruit juice, I’ve heard help you lose weight, I can have that as a routine, right?" Another question asked is "Is it ok to consume milk, eggs, meat, can this be done liberally".

Questions of this nature prompted our team to determine dietary perceptions amongst adults with type 2 diabetes to identify areas requiring emphasis during diet teaching. We used questionnaire based interviews to collect perspectives from 400 participants attending the diabetes clinic at a private center- the Aga Khan University Hospital (AKUH)- (generally speaking, representing high income sector) and public hospital-National Institute Diabetes and Endocrinology (NIDE)- (generally speaking, representing low income sector Karachi, Pakistan). The questionnaire incorporated both closed and open-ended questions. It was designed with the help of a certified nutritionist and diabetologists involved in the care of the patients, incorporating commonly encountered misconceptions and myths, through experience. All interviews were conducted by the same certified clinical nutritionist, from each institute. Consenting subjects were adults (> 18 yrs of age) with type 2 diabetes, presenting for the first time to the diabetes clinic. Fallacies revealed at the interview were rectified then and there by the diabetes educator conducting it.

The mean age of participant at each Institute was $50 \pm 11$ years. At the AKUH and NIDE, there were $55.5 \%$ and $61 \%$ males, respectively. The mean duration of diabetes was $3 \pm 1$ years [AKUH] and $5 \pm 4$ years [NIDE]. We found several misconceptions existing amongst these individuals, irrespective of their socioeconomic or educational status.

Approximately two thirds of the subjects at both (private and public) centers rightly believed that diabetics could have fruit [1] and that whole fruit was favored versus fruit juice. However, many of the study population subjects remarked that they had in the past been led to believe that it is the juice that contained the vitamins and nutrients essential for health that could be conveniently attained in a gulp.
Grapefruit juice was believed by many to be preventive for diabetes, owing to its bitter taste.

A proportion of subjects (25-30\%) believed certain fruit to be prohibited for diabetics, with mangoes and bananas being most cited as the culprit fruits.

Studies have revealed that fruit, including mangoes and bananas, can indeed be consumed by subjects with diabetes [1,2]. The recommendations suggest a small sized fruit, and for a single fruit to be consumed at a particular time, with an average of two to three fruits permitted in a day for an individual with diabetes. Fruits are replete with fiber, vitamins and minerals which makes them a healthy choice for subjects with diabetes $[3,4]$. However, we must remain cognizant of the fact that fruit has a carbohydrate component that must be accounted for as part of the meal plan in both type 1 and type 2 subjects with diabetes. In laymen terminology, this means that there has got to be a "quota system" on fruit consumption. For instance, one sensible way to avoid the carbohydrate load on the body system is to space the fruit from the main meal, and to have a small to medium sized fruit at a time.

Muraki et al. in a prospective longitudinal cohort study sought to determine whether fruit consumption was associated with risk of type 2 diabetes [1]. The study concluded that a greater consumption of specific whole fruits, particularly blue berries, grapes, and apple were associated with a lower incidence of diabetes. Conversely, greater consumption of fruit juice, including grapefruit juice, was associated with a higher risk of diabetes.

*Correspondence to: Azra Rizwan, Aga Khan University Hospital [AKUH], Pakistan, Tel: 9221 34864574; Fax: 9221 493-4294/493-2095; E-mail: azra. rizwan@aku.edu

Received: October 29, 2020; Accepted: November 16, 2020; Published: November 20,2020 
While most work has been done on temperate fruit, little focus had been placed on tropical fruit in terms of how they modulate cellular processes. Some preliminary results suggested that certain components in the mango acted on the same pathways that medication for diabetes and cholesterol targeted. Wilkinson et al. demonstrated the presence of bioactive compounds within the flesh and peel of the mango fruit despite a significant fruit-to-fruit variation [2]. Their work reiterated that eating all components of the fruit was likely to be more beneficial for the body, as compared to refined extracts. This was likely due to depletion of potential antioxidants resulting from the purification process $[2,5]$.

Moving on to dairy products, the egg has been an item of intense debate over the years, in connection with the development of coronary heart disease and type 2 diabetes. Although a food item with high biological value, owing to its rich protein, vitamin and mineral content, its high cholesterol content has traditionally cautioned health care providers against recommending eggs to all individuals, irrespective of individual risk of cardiovascular disease. Studies have attempted to elucidate the connection between dietary cholesterol and cholesterol levels in the blood. Some randomized controlled trials reported that egg intake did not significantly alter blood cholesterol levels, in conflict with previous data [6-8]. A meta-analysis from 2013 reviewed the relationship between egg consumption and risk of cardiovascular diseases and type 2 diabetes, analyzing prospective cohort studies [9]. Their findings suggested that greater egg consumption was not linked with increased overall risk of ischemic heart disease, stroke, or death. However, greater consumption did lead to $42 \%$ greater likelihood of developing diabetes. The authors concluded that regular egg consumption does not enhance the risk of cardiovascular disease and mortality in the general population. Greater egg consumption, however, may be associated with increased likelihood of developing diabetes, and complications of the disorder. The authors surmised that although the mechanisms of this association are not clear at this time, subjects with diabetes, being at high risk for development of cardiovascular disease, may be more sensitive to egg consumption. Consequently, a small increase in circulating cholesterol may lead to a significant increase in risk of coronary heart disease development in subjects with diabetes [9]. A current meta-analysis of prospective cohort studies (2020), in which participants with no baseline history of cardiovascular disease or type 2 diabetes, had been followed for 32 years, revealed that moderate egg consumption was not associated with increased incident cardiovascular disease overall. In the Asian populations there was a potentially lower cardiovascular risk [10].

In our study, almost $50 \%$ of participants from both centers did not know whether a person with diabetes could consume eggs. The dietary advice given by the diabetes educator at both centers emphasized that the egg could be consumed in moderation, that is, three days a week, in line with dietary guidelines on cholesterol intake $[3,4]$ of that time. These guidelines in the light of the most recent evidence [10] would need to be modified with allowance of up to one egg per day.

As regards meat, a vast majority of individuals: $90 \%$ private; $93 \%$ public, stated that any type of meat would be appropriate for the diabetic, versus white meat that has traditionally been recommended. Dietary guidelines have emphasized a restriction on intake of saturated fat, not only refined carbohydrate as an important concept to understand towards the prevention of cardiovascular disease in these high-risk subjects. Results from 2 prospective cohort studies, using repeated measurement of diet, indicated increased mortality from cardiovascular disease and cancer with greater consumption of red meat, owing to its high content of saturated fat [11]. Replacement with servings of fish and poultry were associated with a lower mortality risk. On the other hand, a more recent large epidemiologic cohort study enrolling adult individuals in 18 countries showed no adverse association between saturated fatty acid and cardiovascular disease mortality, having adjusted for confounders, including history of diabetes [12]. The authors have called for reconsideration of guidelines recommending dietary fat restriction for primary prevention of cardiovascular disease in the light of their observations, as well as earlier work indicating similar associations $[13,14]$.

Our study revealed that $68 \%$ of participants at the private sector, while only $45 \%$ from the public center were aware of the importance of whole grain consumption, versus refined grain. This is in keeping with the large epidemiological study mentioned above, which showed that participants from all epidemiological regions, particularly the low income countries, consumed a diet rich in carbohydrates, predominantly those derived from such refined sources as white rice and white bread [11]. Their study reiterated that greater carbohydrate consumption impacted total mortality adversely. What was enlightening was that a lower risk of stroke was found when carbohydrate was replaced with saturated fatty acids [11].

In our study $63 \%$ of participants from the private sector, while $50 \%$ from public sector were unsure of whether greater potato consumption was associated with worsening of blood sugar control. Recent data from 3 cohorts included male and female health professionals, without diabetes and cardiovascular disease at baseline. Participants' fouryear change in potato consumption were calculated using serial food frequency questionnaires. The study revealed that a higher potato consumption was significantly associated with a greater risk of type 2 diabetes [15]. This held true for potatoes cooked in all forms- baked, mashed, boiled or fried, though the risk was greatest for the fried form. This relationship occurred independent of BMI and other risk factors. Furthermore, replacement of potatoes with whole grains was associated with a lower risk of incident diabetes [15].

To top this off with the uncertain impact in the minds of many that milk and yoghurt consumption may have on glycemic control, an average of $31 \%$ at both institutes were not aware of whether or not such dairy products could be consumed. A recent meta-analysis from several observational and randomized controlled trials assessing dairy intake and risk of such chronic disease as diabetes, obesity and cardiovascular disease, concluded that dairy intake may protect against such conditions [16]. They elaborate that whey protein, the principle ingredient of milk and yoghurt, can lead to a reduction in postprandial glucose levels in subjects with type 2 diabetes. This effect may be particularly related to the branched amino acid leucine which has been demonstrated to stimulate the production of glucose- dependent insulitropic polypeptide (GIP) $[16,17]$. A favorable effect of dairy ingredients on insulin sensitivity has also been published in the literature [18]. In keeping with these findings, as well as current dietary recommendations $[3,4]$, the diabetes educator at both institutes encouraged the newly diagnosed subjects with diabetes to consume 2 servings of milk on a daily basis as well as a liberal amount of unsweetened yoghurt.

To conclude, nutrition is an important ingredient for the healthy functioning of the mind and body. Although the rapid advancement in nutrition science has led to some confusion, it has also provided insights into an improved management of such chronic disease as diabetes. Diabetes dietary control goes beyond the myth, still existing in the minds of many, that all one requires is to avoid all sweet food 
items to control blood sugars. Our plea to health care professionals is to keep themselves abreast with on-going research, and consider evidence resulting from such work, particularly regarding fruit, refined carbohydrate and fat consumption in subjects with diabetes. This may help to bridge the knowledge gap regarding dietary modification in these people. Further to this, this may help to remove myths and unnecessary dietary prohibitions placed on these already psychologically stressed individuals, with the tag of this metabolic aberration of dysglycemia, shaping their entire lives.

\section{References}

1. Muraki I, Imamura F, Manson JE, B Hu Frank, Willet W, et al. (2013) Fruit consumption and risk of type 2 diabetes: results from 3 prospective longitudinal cohort studies. $B M J$ Research 347: f5001

2. Wilkinson AS, Flanagan BM, Pierson JT, Hewavitharana AK, Dietzgen RG, et al. (2011) Bioactivity of mango flesh and peel extracts on peroxisome-activated receptor $\gamma$ [PPAR $\gamma$ ] activation and MCF-7 cell proliferation; fraction and fruit variability. J Food Sci 76. [Crossref]

3. American Diabetes Association (2018) Lifestyle management: Standards of medical care in diabetes- 2018. Diabetes Care 41: S42.

4. Mozaffarian D (2016) Dietary and policy priorities for CVD, diabetes and obesity: a comprehensive review. Circulation 133: 187-225. [Crossref]

5. Taing MW, Pierson JT, Hoang VL, Shaw PN, Dietzgen RG, et al. (2012) Mango fruit peel and flesh extracts affect adipogenesis in 3T3-L1 cells. Food Function 3: 828-836. [Crossref]

6. Oh K, Hu FB, Manson JE, Stampfer MJ, Willett WC (2005) Dietary fat intake and risk of coronary heart disease in women: 20 years of follow-up of the Nurses' Health Study. Am J Epidemiol 161: 672-679. [Crossref]

7. Xu J, Eilat-Adar S, Loria C, Goldbourt U, Howard BV, et al. (2006) Dietary fat intake and risk of coronary heart disease: The strong heart study. Am J Clin Nutr 84: 894-902. [Crossref]

8. Skeaff CM, Miller J (2009) Dietary fat and coronary heart disease: summary of evidence from prospective cohort and randomized controlled trials. Ann Nutr Metab 55: 173-201. [Crossref]
9. Shin JY, Xun P, Nakamura Y, He K (2013) Egg consumption in relation to risk of cardiovascular disease and diabetes: a systematic review and meta-analysis. Am J Clin Nutr 98: 146-159. [Crossref]

10. Drouin-Chartier JP, Chen S, Yanping L (2020) Egg Consumption and risk of cardiovascular disease: three large prospective US cohort studies, systematic review, and updated meta-analysis. BMJ 368: $\mathrm{m} 513$. [Crossref]

11. Pan A, Sun Q, Bernstein AM, Schulze MB, Manson JE, et al. (2012) Red meat consumption and mortality: Results from 2 prospective cohort studies. Arch Intern Med 172: 555-563. [Crossref]

12. Dehghan M, Mente A, Zhang X, Swaminathan S, Li W, et al. (2017) Association of fats and carbohydrate intake with cardiovascular disease and mortality in 18 countries from five continents [PURE]: A Prospective cohort study. Lancet 390: 2050-2062. [Crossref]

13. de Souza J, Andrew M, Adriana M, Adrian IC, Vanessa H, et al. (2015) Intake of saturated and trans unsaturated fatty acids and risk of all-cause mortality, cardiovascular disease, and type 2 diabetes: systematic review and meta-analysis of observational studies. BMJ 351: h3978. [Crossref]

14. Ramsden CE, Zamora D, Majchrzak-Hong S, Faurot Keturah R, Broste Steven K, et al (2016) Re-evaluation of the traditional diet-heart hypothesis: analysis of recovered data from Minnsota Coronary Experiment (1968-73). BMJ 353: i1246. [Crossref]

15. Isao M, Rimm EB, Willet WC, Manson JE, Hu FB, et al. (2016) Potato consumption and risk of type 2 diabetes. Results from 3 prospective cohort studies. Diabetes Care 39: 376-384.

16. Thorning TK, Raben A, Tholostrop T, Soedamah-Muthu SS, Givens I, et al. (2016) Milk and dairy products: good or bad for human health? An assessment of the totality of scientific evidence. Food and Nutrition Research 60: 32527. [Crossref]

17. Friad AH, Nilsson M, Holst JJ, Bjorck IM (2005) Effect of whey on blood glucose and insulin responses to composite breakfast and lunch meals in type 2 diabetic subjects. Am J Clin Nutri 82: 69-75. [Crossref]

18. Rideout TC, Marianangeli CP, Martin H, Browne RW, Rempel CB (2013) Consumption of low fat dairy products for 6 months improves insulin resistance without adversely affecting lipids or body weight in healthy adults: a randomized free-living cross-over study. Nutr J 12: 56.

Copyright: (C2020 Rizwan A. This is an open-access article distributed under the terms of the Creative Commons Attribution License, which permits unrestricted use, distribution, and reproduction in any medium, provided the original author and source are credited. 